\title{
Cross-sectional validity of the EQ-5D-Y as a generic health outcome instrument in children and adolescents with cystic fibrosis in
} Germany Daniela Eidt-Koch*1,2, Thomas Mittendorf1,3 and Wolfgang Greiner ${ }^{2}$

Address: ${ }^{1}$ Centre for Health Economics and Health System Research, Leibniz University Hannover, Germany, ${ }^{2}$ Health Economics and Health Care Management, University of Bielefeld, Germany and 3herescon gmbh, Hannover, Germany

Email: Daniela Eidt-Koch* - de@ivbl.uni-hannover.de; Thomas Mittendorf - tm@ivbl.uni-hannover.de; Wolfgang Greiner - wolfgang.greiner@uni-bielefeld.de

* Corresponding author

Published: 28 August 2009

BMC Pediatrics 2009, 9:55 doi:10.1186/147|-243|-9-55
Received: 21 August 2008

Accepted: 28 August 2009

This article is available from: http://www.biomedcentral.com//47/-243 I/9/55

(C) 2009 Eidt-Koch et al; licensee BioMed Central Ltd.

This is an Open Access article distributed under the terms of the Creative Commons Attribution License (http://creativecommons.org/licenses/by/2.0), which permits unrestricted use, distribution, and reproduction in any medium, provided the original work is properly cited.

\begin{abstract}
Background: Quality of life is recognized as an important additional outcome measure in clinical trials and health economic evaluations. The EQ-5D is an important generic health outcome instrument often used for economic evaluations as a complement with disease-specific outcome measures. In this study quality of life data was assessed using the EQ-5D-Y (new EQ-5D version for children and adolescents) and the Cystic Fibrosis Questionnaire (CFQ). The objective of the study is to evaluate the cross-sectional validity of the EQ-5D-Y as a generic health outcome instrument in children and adolescents with cystic fibrosis in Germany.
\end{abstract}

Methods: In 2006 a multi-centre study was conducted in four cystic fibrosis centres in Germany. Quality of life data from 96 patients between eight and seventeen years was collected using the EQ5D-Y as a generic outcome instrument and the Cystic Fibrosis Questionnaire (CFQ) as a diseasespecific instrument. Results of both instruments were compared by statistical analyses using Spearman's rank correlations.

Results: $44.6 \%$ of the patients stated that they had no problems in any of the EQ-5D-Y dimensions. Several low to high correlations between separate dimensions and the visual analogue scale of the EQ-5D-Y and the different scales of the CFQ for children, their parents and adolescents can be presented in this paper. Looking at the five EQ-5D-Y dimensions the highest correlation $\left(r_{S}=0.625\right.$, $P=0.0 \mathrm{I}$ ) was found between the dimension 'mobility' and the CFQ scale 'physical functioning' in adolescent patients. The overall highest correlation was found between the 'subjective health perception' and the visual analogue scale $\left(r_{s}=0.744, p=0.01\right)$ in adolescent patients older than I3 years.

Conclusion: The EQ-5D-Y can be considered a cross-sectional valid generic health outcome instrument which reflects differences in health according to the progression of the life-long chronic disease cystic fibrosis. 


\section{Background}

Quality of life is recognized as an important additional outcome measure in clinical trials and health economic evaluations. In the past two decades many instruments for the assessment of quality of life have been developed. Most of the developed instruments for the assessment of quality of life are for adults. However, quality of life instruments for children, which are applicable in healthy as well as chronically ill children, are important and their use in clinical research should be expanded [1].

The EQ-5D is an important generic health outcome instrument often used for economic evaluations as a complement with disease-specific outcome measures. As of now, the EQ-5D is only validated for adults. Therefore the internationally applicable child friendly version EQ-5D-Y has been established by the EuroQol group and translated into many different languages (Wille N, the EuroQol Youth Task Force \& Ravens-Sieberer U: Development of a child-friendly EQ-5D: the EQ-5D-Y international version, submitted). In this multi centre study quality of life data from cystic fibrosis children and adolescents was assessed using the EQ-5D-Y as a generic health outcome instrument and the Cystic Fibrosis Questionnaire (CFQ) as a disease-specific instrument in cystic fibrosis patients.

Cystic fibrosis is a complex lifelong chronic disease caused by genetic mutations. In most cases multiple organ systems are affected, most patients especially suffer from pancreatic insufficiency and lung function problems. Hence, this chronic condition leads to irreversible organ damages with patients having a life expectancy of only 29 years in 2007 [2].

In this article the results of the EQ-5D-Y as well as the results of the CFQ will be analysed and correlations between dimensions and scales of the instruments will be demonstrated. The aim is to assess the cross-sectional validity of the EQ-5D-Y.

\section{Methods}

The multi-centre study was based on single cross-sectional data collection from children and adolescents between 8 to 17 years with cystic fibrosis in continuous ambulatory treatment in four German cystic fibrosis centres (Hannover Medical School, Johann Wolfgang Goethe University Hospital, Children's Hospital, University of Heidelberg, and the Dr. von Haunersches Kinderspital, Ludwig Maximilian University Munich) between April and August 2006. The study has been approved by the ethic committees of the involved clinics. The patients gave their consent for the study.
Two instruments were used to collect quality of life data: the EQ-5D-Y as a generic health outcome instrument and the CFQ as a disease-specific instrument.

The EQ-5D consists of five dimensions of health: mobility, self-care, usual activities, pain/discomfort and anxiety/ depression. The respondent can choose the levels no (1), some (2) or extreme (3) problems. The result is a health profile, e.g. a patient with the health profile 11223 has no problems with mobility and self-care, some problems with everyday activities and pain/discomfort and extreme problems with happiness/worry/sadness, respectively. The visual analogue scale (VAS) included in the EQ-5D is used to capture the patients subjective health perception between worst imaginable health state (score 0 ) and best imaginable health state (score 100) [3]. The EQ-5D was initially constructed and validated for adult patients. The EuroQol group now created a version for children in different languages. The EQ-5D-Y differs from the adult version in changes of words which especially were adapted for children. The German child-friendly version of the EQ5D (EQ-5D-Y) is available at the EuroQol group http:// www.euroqol.org. First studies about the child-friendly EQ-5D were presented on the $23^{\text {rd }}$ Scientific Plenary Meeting of the EuroQol Group in Barcelona, Spain, September 14-16, 2006 [4-10].

The Cystic Fibrosis Questionnaire (CFQ) is a disease-specific instrument for quality of life measurement in cystic fibrosis patients developed in France. Translations and validations for German language are available [11]. The CFQ has been developed in different versions for children from 8 to 13 years (CFQ-k) and their parents (CFQ-e) as well as a version for adolescents and adults aged 14 years or older (CFQ 14+). The CFQ consists of 35 (CFQ-k), 44 (CFQ-e) or 50 (CFQ 14+) items containing different modules (quality of life, general health perception, symptoms), dimensions and scales, respectively. Scales in the generic dimensions of quality of life are physical functioning, energy, emotional state, social limitations, everyday life/school problems, in the disease specific dimensions body image, eating disturbance and treatment burden. Scales for the symptoms are weight problems, respiratory and digestive symptoms. Answers of the patients are transformed into scales between 0 (worst) and 100 (best).

According to similar concepts and content of the questionnaires high correlations $\left(\mathrm{r}_{\mathrm{S}}>0.5, \mathrm{p}<0.01\right)$ are especially hypothesized for example between the EQ-5D-Y dimension 'mobility' and the CFQ dimension 'physical functioning', between the EQ-5D-Y dimension 'happiness/worry/sadness' and the CFQ dimension 'emotional state' as well as between the EQ-5D-Y VAS and the CFQ dimension 'subjective health perception'. Moderate $(0.3 \leq$ $\left.r_{S} \leq 0,5\right)$ or mild correlations $\left(r_{S}<0.3\right)$ are hypothesized 
for example between the EQ-5D-Y dimension 'pain/discomfort' and the and the CFQ scales 'respiratory symptoms' and 'digestive symptoms' as well as the EQ-5D-Y VAS and the CFQ scales 'physical functioning' and 'emotional state' could be expected for. According to different issues no significant correlations are for example hypothesized between the EQ-5D-Y dimension 'self care' and especially for the CFQ scales 'body image'.

A subgroup analysis was made for patients with no problems on EQ-5D-Y (health profile 11111).

Statistical analyses in this paper were performed using SPSS and EXCEL.

\section{Results}

Socio-demographic and clinical data

Data from 96 patients with an age between 8 and 17 years were collected. 55 patients (57.3\%) were between 8 and 13 years (mean age: 10.8 years) and 41 patients (42.7\%) were between 14 and 18 years old (mean age: 15.9 years). Patients in this multi-centre study were treated in the ambulatory cystic fibrosis centres of the Hanover Medical
School (22 patients), Johann Wolfgang Goethe University Hospital (24 patients), Children's Hospital, University of Heidelberg (5 patients), and the Dr. von Haunersches Kinderspital, Ludwig Maximilian University Munich (45 patients). Clinical data is shown in table 1.

Lung function of these patients is worse than normal levels especially for $\mathrm{MEF}_{25}$. In addition, the clinical data shows, that $63.6 \%$ of all children and $73.2 \%$ of the adolescents have a bacterial colonization of their lungs. About $80 \%$ of the patients have a pancreatic insufficiency. As expected adolescents (with a longer disease history than children) have a worse health status than those of lower age. A significant difference between age groups can be found especially in patients having a bacterial colonization of the lungs (Mann-Whitney-U-Test, $\mathrm{p}=0.011$ ).

\section{EQ-5D-Y dimensions and visual analogue scale (VAS)}

Figure 1 presents the distribution within the EQ-5D-Y dimensions. Between $84 \%$ and $89 \%$ of the patients reported no problems (score 1) with 'mobility', 'self-care' and 'usual activities'. $62 \%$ to $65 \%$ of the patients experienced no problems with 'pain/discomfort' or 'happiness/

Table I: Clinical data

\begin{tabular}{|c|c|c|c|}
\hline Variable & $\begin{array}{c}\text { Children } \\
\text { (8 to I3 years) } \\
(n=55)\end{array}$ & $\begin{array}{c}\text { Adolescents } \\
\text { (I4 to I } 7 \text { years) } \\
(n=4 I)\end{array}$ & Significance level \\
\hline Sex (male) & $43.6 \%(n=24)$ & $58.5 \%(n=24)$ & \\
\hline Age (mean/SD) & $10.8 / 1.7$ & $15.9 / 1.80$ & \\
\hline \% vital capacity (mean/SD) & $92.5 \% / 11.9 \%(n=47)$ & $97.2 \% / 13.1 \%(n=34)$ & $P=0.082$ \\
\hline$\% \mathrm{FEV}_{\mathrm{I}}(\mathrm{mean} / \mathrm{SD})$ & $93.6 \% / 15.2 \%(n=47)$ & $90.7 \% / 20.3 \%(n=34)$ & $P=0.618$ \\
\hline$\% \mathrm{MEF}_{25}($ mean/SD $)$ & $68.4 \% / 41.7 \%(n=47)$ & $58.9 \% / 37.5 \%(n=34)$ & $p=0.273$ \\
\hline Bacterial colonization of the lung & $63.6 \%(n=35)$ & $73.2 \%(n=30)$ & $P=0.009$ \\
\hline Pneumothorax & $1.8 \%(n=1)$ & $0 \%(n=0)$ & $p=0.392$ \\
\hline Allergic bronchopulmonary aspergillosis (ABPA) & $3.6 \%(n=2)$ & $12.2 \%(n=5)$ & $P=0.229$ \\
\hline Pancreatic insufficiency & $80.0 \%(n=44)$ & $78.1 \%(n=32)$ & $P=0.782$ \\
\hline Hepatobiliary complications & $23.6 \%(n=13)$ & $26.8 \%(n=11)$ & $P=0.840$ \\
\hline Distal intestinal obstruction & $7.3 \%(n=4)$ & $0.0 \%(n=0)$ & $p=0.082$ \\
\hline Diabetes mellitus & $0.0 \%(n=0)$ & $7.3 \%(n=3)$ & $p=0.041$ \\
\hline Nasal polyp & $10.9 \%(n=6)$ & $17.1 \%(n=7)$ & $P=0.366$ \\
\hline Isolation obligation for patient & $1.8 \%(n=1)$ & $9.8 \%(n=4)$ & $P=0.081$ \\
\hline
\end{tabular}




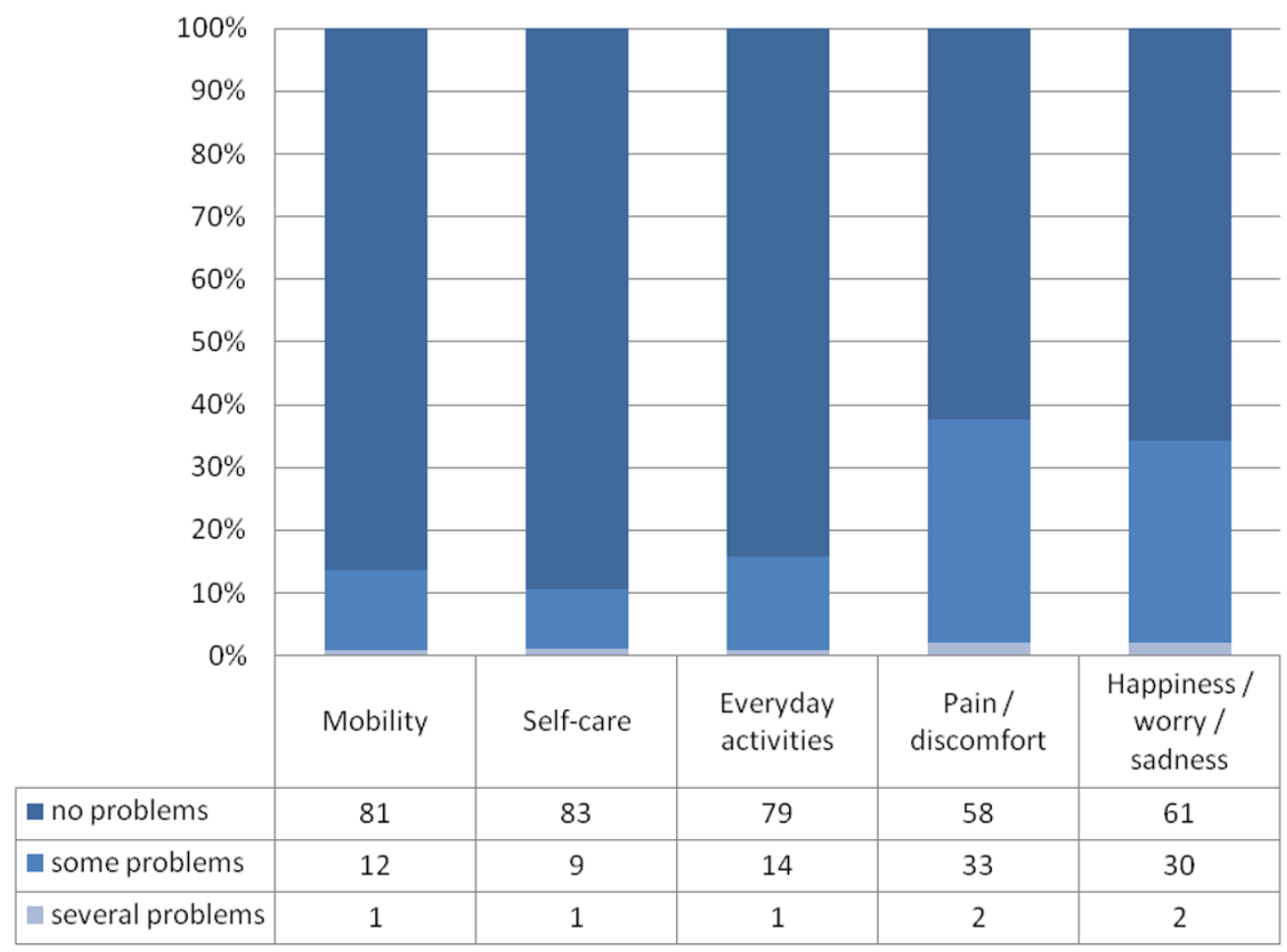

Figure I

Distribution of EQ-5D-Y dimensions ( $n=94)$.

worry/sadness'. Only one or two patients reported extreme problems (score 3) on at least one EQ-5D-Y dimension.

92 patients had no missing data. Health states were reported from health state 11111 (no problems in all dimensions, $\mathrm{n}=41,44.6 \%$ ) to health state 22233 (some problems with 'mobility', 'self-care' and 'usual activities' and extreme problems with 'pain/discomfort', $\mathrm{n}=1$, $1.1 \%)$. The high proportion of patients with no problems hints at a ceiling effect typical for a generic instrument like the EQ-5D-Y. However, additional analyses showed significant differences in all but one CFQ subscale ('digestive symptoms, Mann-Whitney-U-Test, $\mathrm{p}=0.08$ ) for patients with any problem in comparison to patients without any problems with respect to the EQ-5D-Y. Furthermore, an subgroup analysis for patients who reported no problems on the EQ-5D-Y resulted in no significant differences in all CFQ subscales for patients with different clinical status, for example in pancreatic insufficiency or bacterial colonization of the lungs see table 2 .

As a result the EQ-5D-Y showed to be able to discriminate problems in different areas.
90 patients filled out the visual analogue scale about their subjective health perception. The mean VAS for eight to 13-year old patients was 85.4 (SD 16.4), the mean VAS for 14 to 17 year old patients was 79.4 (SD 13.2). Hence, the VAS value is significantly better for younger patients than for older patients (Mann-Whitney-U-Test, $\mathrm{p}=0.006$ ).

\section{Correlations between EQ-5D-Y and CFQ}

93 patients completed the disease-specific CFQ. 55 patients between 8 and 13 years as well as their parents reported quality of life by the CFQ-k (for children) and the CFQ-e (for parents). 38 adolescent patients reported their quality of life by the CFQ 14+ (for patients older than 14 years).

Correlations between EQ-5D-Y dimensions and the CFQ scales are reported in table 3 . If the correlation (Spearman's rank correlation) is significant at a significance level of 0.01 it is marked with two stars $\left({ }^{*}\right)$. One star $\left({ }^{*}\right)$ indicates that the correlation is significant at a level of 0.05 (non-significant correlations are reported as n.s.).

For all CFQ versions the EQ-5D-Y dimension 'mobility' correlates moderately $\left(0.3 \leq \mathrm{r}_{\mathrm{S}} \leq 0.5, \mathrm{p}<0.01\right)$ or highly $\left(\mathrm{r}_{\mathrm{S}}>0.5, \mathrm{p}<0.01\right)$ with the scale 'physical functioning'. For the dimension 'self care' several mild $\left(\mathrm{r}_{\mathrm{S}}<0.3, \mathrm{p}<\right.$ 
Table 2: Subgroup analysis for patients without problems on EQ-5D-Y

\begin{tabular}{|c|c|c|c|c|}
\hline & \multicolumn{2}{|l|}{ Pancreatic insufficiency $(\mathrm{PI})$} & \multicolumn{2}{|c|}{ Bacterial colonization (BC) } \\
\hline & Mean without $\mathrm{PI}(\mathrm{SD}) /$ Mean with $\mathrm{PI}(\mathrm{SD})$ & Significance level & $\begin{array}{l}\text { Mean without } B C(S D) / \text { Mean with } B C \\
\text { (SD) }\end{array}$ & Significance level \\
\hline Physical functioning & $91.7(14.0) / 92.2(12.4)$ & $P=0.985$ & $95.9(4.7) / 89.4(15.2)$ & $P=0.337$ \\
\hline Emotional state & $85.5(10.9) / 87.5(10.4)$ & $p=0.616$ & $88.1(11.5) / 86.1(9.7)$ & $p=0.408$ \\
\hline Social limitations & $66.4(14.4) / 72.6(11.2)$ & $P=0.143$ & $73.1(12.3) / 69.7(11.3)$ & $p=0.469$ \\
\hline Body image & $79.4(17.5) / 87.5(20.0)$ & $P=0.153$ & $85.2(24.7) / 87.5(16.2)$ & $P=0.987$ \\
\hline Eating disturbance & $88.9(17.0) / 94.8(9.8)$ & $p=0.344$ & 91.9 (12.9)/94.7 (10.6) & $p=0.376$ \\
\hline Treatment burden & $77.8(17.0) / 74.0(19.3)$ & $P=0.765$ & $68.1(20.1) / 77.8(16.8)$ & $p=0.135$ \\
\hline Respiratory symptoms & $82.1(11.7) / 86.0(16.2)$ & $p=0.212$ & $88.0(9.3) / 83.1(18.2)$ & $p=0.509$ \\
\hline Digestive symptoms & $76.2(16.3) / 78.5(20.1)$ & $P=0.613$ & $75.6(19.3) / 80.2(19.8)$ & $P=0.457$ \\
\hline
\end{tabular}

$0.01)$ or moderate $\left(0.3 \leq \mathrm{r}_{\mathrm{S}} \leq 0.5, \mathrm{p}<0.01\right)$ correlations with CFQ scales can be found, but overall the correlations of this dimension are the lowest in comparison to the other dimensions. The dimension 'everyday activities' shows several moderate correlations especially with the scales 'physical functioning', 'emotional state', 'everyday life' and 'treatment burden'. Some CFQ scales correlate highly to the EQ-5D-Y dimension 'pain/discomfort'. For the dimension 'happiness/worry/sadness' the highest correlation $\left(\mathrm{r}_{\mathrm{S}}=0.586, \mathrm{p}=0.01\right)$ can be found with the CFQ scale 'emotional state' in adolescent patients.

For all CFQ versions the visual analogue scale shows moderate or high correlations $\left(\mathrm{r}_{\mathrm{s}}>0.3, \mathrm{p}<0.01\right)$ with the CFQ scales 'physical functioning', 'energy', 'school problems' and 'everyday life', 'subjective health perception', and 'respiratory symptoms'. The overall highest correlation $\left(r_{S}=\right.$ $0.744, \mathrm{p}=0.01$ ) can be found between 'subjective health perception' and the visual analogue scale in adolescent patients.

Only a few statistically significant correlations between clinical data and quality of life data can be found. The dimension 'usual activities' shows low correlations with the '\% of vital capacity' $\left(\mathrm{r}_{\mathrm{S}}=0.248, \mathrm{p}<0.05\right)$ and if a patient has to be isolated according to a bacterial colonization of his lungs $\left(r_{S}=0.281, p<0.01\right)$. The visual analogue scale correlates moderately especially with age $\left(\mathrm{r}_{\mathrm{S}}=\right.$ $0.339, \mathrm{p}<0.01)$ and weakly with a bacterial colonization of the lungs $\left(r_{S}=0.215, p<0.05\right)$. Therefore differences in clinical data seem not to have a major impact on quality of life data.

\section{Discussion}

Summary of the results

$44.6 \%$ of the patients stated that they had no problems in any of the EQ-5D-Y dimensions. The high proportion of patients with no problems hints at a ceiling effect typical for a generic instrument like the EQ-5D-Y. However, additional analyses showed that the EQ-5D-Y is able to discriminate problems in different areas.

Several low to strong correlations between the dimensions of the EQ-5D-Y and the scales of the CFQ for children, their parents and adolescents could be found in the analysis. Looking at the five EQ-5D-Y dimensions the highest correlation $\left(r_{S}=0.625, p=0.01\right)$ was found between the dimension 'mobility' and the CFQ scale 'physical functioning' in adolescent patients. For all CFQ versions the visual analogue scale showed moderate or high correlations $\left(\mathrm{r}_{\mathrm{S}}>0.3\right)$ with several CFQ scales. The overall highest correlation was found between the 'subjective health perception' and the visual analogue scale $\left(\mathrm{r}_{\mathrm{S}}=0.744, \mathrm{p}=0.01\right)$ in adolescents. Hence, the hypothesized associations were confirmed.

Only a few correlations between clinical data and quality of life data were found. Therefore, differences in clinical data may not have a major impact on quality of life data for cystic fibrosis children and adolescents.

\section{Interpretation of the results}

It is noticeable that in many cases patients reported about a good EQ-5D health state despite their strong disease according to the medical classification of disease activity. The objective health state may be actually lower (worse) 
Table 3: Correlations between EQ-5D-Y dimensions and CFQ scales

\begin{tabular}{|c|c|c|c|c|c|c|c|}
\hline & & Mobility & Self care & Everyday activities & Pain/discomfort & Happiness/worry/sadness & VAS \\
\hline \multirow[t]{3}{*}{ Physical functioning } & CFQk & $-0.459(* *)$ & $-0.309(*)$ & $-0.379(* *)$ & $-0.419(* *)$ & $-0.299(*)$ & $0.401(* *)$ \\
\hline & CFQe & $-0.516(* *)$ & $-0.307(*)$ & $-0.438(* *)$ & $-0.417(* *)$ & -0.267 (n.s.) & $0.495(* *)$ \\
\hline & CFQ 14+ & $-0.625(* *)$ & $-0.495(* *)$ & $-0.472(* *)$ & -0.247 (n.s.) & -0.149 (n.s.) & $0.576(* *)$ \\
\hline \multirow[t]{2}{*}{ Energy } & CFQe & -0.248 (n.s.) & -0.181 (n.s.) & -0.263 (n.s.) & $-0.509(* *)$ & $-0.315(*)$ & 0.469 (**) $^{* *}$ \\
\hline & CFQ 14+ & -0.248 (n.s.) & $-0.358(* *)$ & -0.266 (n.s.) & $-0.408(* *)$ & $-0.396(* *)$ & $0.726(* *)$ \\
\hline \multirow[t]{3}{*}{ Emotional state } & CFQk & -0.188 (n.s.) & -0.096 (n.s.) & $-0.286(*)$ & $-0.507(* *)$ & $-0.345(*)$ & $0.308\left(^{*}\right)$ \\
\hline & CFQe & $-0.428(* *)$ & $-0.317(*)$ & $-0.402(* *)$ & $-0.517(* *)$ & $-0.421(* *)$ & $0.445\left(^{* *}\right)$ \\
\hline & CFQ 14+ & $-0.337(* *)$ & $-0.451(* *)$ & $-0.373(* *)$ & $-0.433(* *)$ & $-0.586(* *)$ & $0.567(* *)$ \\
\hline \multirow[t]{2}{*}{ Social limitations } & CFQk & $-0.338(*)$ & -0.201 (n.s.) & -0.255 (n.s.) & $-0.350(* *)$ & $-0.314(*)$ & 0.277 (n.s.) \\
\hline & CFQ 14+ & -0.255 (n.s.) & -0.200 (n.s.) & -0.309 (n.s.) & -0.315 (n.s.) & 0.030 (n.s.) & $0.435(* *)$ \\
\hline School problems & CFQe & $-0.316\left(^{*}\right)$ & -0.268 (n.s.) & $-0.327(*)$ & $-0.519\left(^{* *}\right)$ & $-0.362(* *)$ & $0.452(* *)$ \\
\hline Everyday life & CFQ 14+ & $-0.410(* *)$ & $-0.365(* *)$ & $-0.461(* *)$ & $-0.599(* *)$ & $-0.412(* *)$ & $0.553(* *)$ \\
\hline \multirow[t]{3}{*}{ Body image } & CFQk & -0.171 (n.s.) & -0.178 (n.s.) & $-0.292(*)$ & $-0.293(*)$ & $-0.391(* *)$ & $0.347\left(^{*}\right)$ \\
\hline & CFQe & -0.180 (n.s.) & -0.234 (n.s.) & -0.234 (n.s). & $-0.279(*)$ & -0.265 (n.s.) & $0.327\left(^{*}\right)$ \\
\hline & CFQ I4+ & -0.139 (n.s.) & $-0.427(*)$ & $-0.459(* *)$ & $-0.425(* *)$ & -0.235 (n.s.) & $0.551(* *)$ \\
\hline \multirow[t]{3}{*}{ Eating disturbance } & CFQk & $-0.294(*)$ & $-0.338(*)$ & $-0.343(*)$ & $-0.311(*)$ & -0.186 (n.s.) & 0.187 (n.s.) \\
\hline & CFQe & $-0.286\left(^{*}\right)$ & -0.253 (n.s.) & -0.175 (n.s.) & -0.139 (n.s.) & 0.032 (n.s.) & 0.170 (n.s.) \\
\hline & CFQ I4+ & $-0.367(* *)$ & $-0.465(* *)$ & $-0.391(* *)$ & -0.174 (n.s.) & -0.256 (n.s.) & 0.315 (n.s.) \\
\hline \multirow[t]{3}{*}{ Treatment burden } & CFQk & -0.155 (n.s.) & -0.168 (n.s.) & $-0.433(* *)$ & $-0.365(* *)$ & $-0.340(*)$ & 0.166 (n.s.) \\
\hline & CFQe & -0.229 (n.s.) & -0.168 (n.s.) & $-0.361(* *)$ & -0.254 (n.s.) & $-0.346(*)$ & 0.315 (n.s.) \\
\hline & CFQ 14+ & -0.298 (n.s.) & $-0.473(* *)$ & -0.282 (n.s.) & -0.217 (n.s.) & -0.320 (n.s.) & $0.439(* *)$ \\
\hline \multirow{2}{*}{$\begin{array}{l}\text { Subjective health } \\
\text { perception }\end{array}$} & CFQe & $-0.336(*)$ & $-0.308(*)$ & $-0.380(* *)$ & $-0.508\left(^{* *}\right)$ & $-0.364(* *)$ & $0.379(* *)$ \\
\hline & CFQ I4+ & $-0.435(* *)$ & $-0.357(* *)$ & $-0.349(* *)$ & $-0.470(* *)$ & $-0.460(* *)$ & $0.744(* *)$ \\
\hline \multirow[t]{2}{*}{ Weight problems } & CFQe & -0.116 (n.s.) & $-0.347(*)$ & -0.123 (n.s.) & $-0.355(* *)$ & -0.129 (n.s.) & 0.266 (n.s) \\
\hline & CFQ 14+ & $-0.374(* *)$ & $-0.495(*)$ & $-0.533(* *)$ & -0.240 (n.s.) & -0.233 (n.s.) & 0.256 (n.s.) \\
\hline \multirow[t]{3}{*}{ Respiratory symptoms } & CFQk & -0.235 (n.s.) & 0.004 (n.s.) & $-0.328(*)$ & $-0.315(*)$ & $-0.304(*)$ & $0.393(* *)$ \\
\hline & CFQe & $-0.434(* *)$ & -0.228 (n.s.) & $-0.383(* *)$ & $-0.376(* *)$ & $-0.302(*)$ & $0.450(* *)$ \\
\hline & CFQ 14+ & $-0.469(* *)$ & -0.258 (n.s.) & -0.286 (n.s.) & $-0,259$ (n.s.) & $-0.457(* *)$ & $0.592(* *)$ \\
\hline
\end{tabular}


Table 3: Correlations between EQ-5D-Y dimensions and CFQ scales (Continued)

\begin{tabular}{|c|c|c|c|c|c|c|c|}
\hline \multirow[t]{3}{*}{ Digestive symptoms } & CFQk & -0.157 (n.s.) & $-0.309\left(^{*}\right)$ & $-0.380(* *)$ & $-0.474\left(^{* *}\right)$ & -0.066 (n.s.) & 0.153 (n.s.) \\
\hline & CFQe & -0.115 (n.s.) & -0.227 (n.s.) & -0.239 (n.s.) & $-0.453(* *)$ & 0.056 (n.s.) & 0.179 (n.s.) \\
\hline & CFQ I4+ & -0.162 (n.s.) & -0.229 (n.s.) & -0.221 (n.s.) & -0.214 (n.s.) & -0.200 (n.s.) & $0.496(* *)$ \\
\hline
\end{tabular}

CFQk = Cystic Fibrosis Questionaire (version for children from 8 to 13 years)

$\mathrm{CFQe}=$ Cystic Fibrosis Questionaire (version for parents of children from 8 to 13 years)

CFQ 14+ = Cystic Fibrosis Questionaire (version for adolescents from 14 to 17 years)

$*=$ significant at $\mathrm{p}<0.01$

$* *=$ significant at $p<0.05$

n.s. $=$ not significant

than reported. An explanation might be coping, which means that patients learn to live with the disease and do not perceive limitations as bad as expected.

Different significance levels for correlations between EQ$5 \mathrm{D}-\mathrm{Y}$ dimensions and CFQ scales might result from the fact, that some dimensions of the EQ-5D-Y and scales of the CFQ are directed at exactly the same issue (for example the visual analogue scale of the EQ-5D-Y and the CFQ scale 'subjective health perception') and others only partially. Furthermore a reason for higher correlations of the visual analogue scale in comparison to the EQ-5D-Y dimensions might be the continuous character of the scale instead of the three score levels.

Although the time frame of the EQ-5D-Y (today) is different to the time frame of the CFQ (last two weeks), significant correlation results were found. However, it is possible that correlations within the same time frame still would be higher than those reported.

For further research it would be interesting to compare different properties of the used measures in a setting with multiple and continuous assessments at various time points.

\section{Conclusion}

Data was collected in a multi centre study from a representative patient group of children and adolescents with cystic fibrosis. The analysis showed several correlations between the different dimensions and the visual analogue scale of the EQ-5D-Y and the scales of the different CFQ versions for children, their parents and adolescents. Therefore, the EQ-5D-Y can be considered a cross-sectional valid generic health outcome measure which reflects differences in health according to the progression of the life long chronic disease cystic fibrosis.

\section{Competing interests}

The authors declare that they have no competing interests.

\section{Authors' contributions}

DE-K collected the data, did the statistical analyses and writing of the manuscript. TM and WG participated in conception, design, and interpretation of data as well as revision of the manuscript. All authors read and approved the final manuscript.

\section{Acknowledgements}

We would like to especially thank the Mukoviszidose e.V., the EuroQol Child-friendly Task Force, Doris Staab (CFQ) as well as the clinics and patients for their support and information they provided for this analysis.

\section{References}

I. Bullinger M, Schmidt S, Petersen C, Ravens-Sieberer U: Quality of life - evaluation criteria for children with chronic conditions in medical care. J Public Health 2006, 14:343-355.

2. Stern M, Sens B, Wiedemann B, Busse O, Damm G, Wenzlaff P, (Eds): Qualitätssicherung Mukoviszidose - Überblick über den Gesundheitszustand der Patienten in Deutschland 2007. Berlin: Medizinisch Wissenschaftliche Verlagsgesellschaft; 2009.

3. Greiner W, Claes C, Busschbach JJ, Schulenburg JM: Validating the EQ-5D with time trade off for the German population. Eur J Health Econ 2005, 2: 124-130.

4. Gusi N, Badía X, Herdman M, Olvares P: Translation and testing of a Spanish Child-Friendly version of EQ-5D and the appropriateness of the EQ-5D. In 23rd Scientific Plenary Meeting of the EuroQol Group in Barcelona, Spain: September 14-16 Edited by: Badía X. Barcelona: IMS Health; 2006:93-I 18.

5. Scalone L, Cavrini G, Pacelli B, Mattivi A: Translation and testing of an Italian Child-Friendly version of EQ-5D. In 23rd Scientific Plenary Meeting of the EuroQol Group in Barcelona, Spain: September 1416 Edited by: Badía X. Barcelona: IMS Health; 2006:I I9-174.

6. Tomasetto C, Matteucci MC, Selleri P, Cavrini G: The Contribution of lexical analyses of the wording in cognitive interviews during the CF-EQ-5D italian translation. In 23rd Scientific Plenary Meeting of the EuroQol Group in Barcelona, Spain: September I 4-16 Edited by: Badía X. Barcelona: IMS Health; 2006:175-215.

7. Wille N, Ravens-Sieberer U: Age-appropriateness of the EQ-5D adult and child-friendly version - Testing the feasibility, reliability and validity in children and adolescents. In 23rd Scientific Plenary Meeting of the EuroQol Group in Barcelona, Spain: September I416 Edited by: Badía X. Barcelona: IMS Health; 2006:217-229.

8. Wille N, Ravens-Sieberer U, the child-friendly task force on behalf of the EuroQol Group: Establishing definitions of the concepts included in CF-EQ-5D - A revision of the "Definition of EQ5D concepts" for adults. In 23rd Scientific Plenary Meeting of the EuroQol Group in Barcelona, Spain: September 14-16 Edited by: Badía X. Barcelona: IMS Health; 2006:233-256.

9. Wille N, Ravens-Sieberer U, on behalf of the EuroQol Group: Proposal on a core set of instruments for validation of the international child-friendly EQ-5D. In 23rd Scientific Plenary Meeting of the EuroQol Group in Barcelona, Spain: September 14-16 Edited by: Badía X. Barcelona: IMS Health; 2006:259-276. 
10. Jelsma J, Coppez P, Jehosofat T, Lenders C: The concurrent validity of the Child Friendly version of the EQ-5D when used with high school English speaking children in South Africa. In 23rd Scientific Plenary Meeting of the EuroQol Group in Barcelona, Spain: September 14-16 Edited by: Badía X. Barcelona: IMS Health; 2006:277-295.

II. Wenninger K, Aussage P, Wahn U, Staab D, the German CF Study Group: The revised German Cystic Fibrosis Questionnaire: Validation of a disease-specific health-related quality of life instrument. Qual Life Res 2003, I 2:77-85.

\section{Pre-publication history}

The pre-publication history for this paper can be accessed here:

http://www.biomedcentral.com/1471-2431/9/55/prepub

Publish with Bio Med Central and every scientist can read your work free of charge

"BioMed Central will be the most significant development for disseminating the results of biomedical research in our lifetime. "

Sir Paul Nurse, Cancer Research UK

Your research papers will be:

- available free of charge to the entire biomedical community

- peer reviewed and published immediately upon acceptance

- cited in PubMed and archived on PubMed Central

- yours - you keep the copyright

Submit your manuscript here:

http://www.biomedcentral.com/info/publishing_adv.asp 